\title{
A Mathematical Model for an Acceleration of a Rotating Object about a Fixed Axis
}

Usubamatov $\mathbf{R}^{*}$, Abdykerimova $\mathrm{D}$ and Dotalieva $\mathbf{Z}$

Department of Mathematics, Kyrgyz State Technical University, Ave, Bishkek, Kyrgyzstan

\begin{abstract}
Kinematics of a rotating object about a fixed axis is well described in science of classical mechanics. All fundamental publications and textbooks contain a mathematical model for an acceleration of a rotating object about a fixed axis that is used for computing of forces acting on an object. Practice of usage for computing an acceleration a rotating object did not show some claims to the known equation.

Nevertheless, the detailed analysis of the mathematical model for kinematics of a rotation of an object with acceleration demonstrates its incorrectness. The value of the actual acceleration of the rotating object is less than computed by the known equation. This is reason that running mechanisms are operating without fails because the value of acting forces is bigger than actual one. It means, the component of mechanisms is stronger and heavier than necessary. This paper addresses an acceleration analysis of a rotating object about a fixed axis and presents the mathematical model for computing an acceleration that can be used in engineering.
\end{abstract}

Keywords: Kinematics; Angular velocity; Acceleration; Rotating object

\section{Introduction}

Machines and mechanisms running at a high-speed are considered by a kinematic analysis of their velocities and accelerations, and subsequently analyzed as a dynamic system in which forces due to accelerations are analyzed using the principles of kinetics [1-3]. Many fundamental methods and approaches exist to find accelerations in classical mechanisms that represented in the textbooks [4-6].

There are numerous publications that consider special cases of kinematics and dynamics of machinery [7-9]. The complex motions of the objects are considered by numerical methods if known analytic approaches cannot give correct solutions $[10,11]$. Science of classical mechanics enables solving all problems of kinematics and dynamics of machinery. Nevertheless, practice of machines and mechanisms demonstrate numerous conditions with increasing the regimes of their work. High velocities and forces acting on machines request high responsibility in computing of their parameters.

This paper represents an acceleration analysis of a rotating object about a fixed axis and comparison with known publications.

The known publications demonstrate that the curvilinear motion of an object with a variable speed is going on with a linear acceleration, which is commonly separated on two elements: tangential and radial components of acceleration. The tangential component is formed as a result of a change in the magnitude of the linear velocity vector and its direction is perpendicular to the line that connects the object with the axis of rotation. The radial component is created as a result of a change in the direction of the linear velocity vector and its direction is always toward the axis of a rotating object. Expression of an absolute acceleration $\alpha$ of an object represents the sum of vectors of the tangential $\boldsymbol{\alpha}_{\boldsymbol{t}}$, and radial $\boldsymbol{\alpha}_{\boldsymbol{r}}$ components. Expression of an absolute acceleration of a rotating object has the following equation [1-9]:

$$
\alpha=\sqrt{a_{t}^{2}+a_{r}^{2}}=r \sqrt{\varepsilon^{2}+\omega^{4}}
$$

where $\alpha=\varepsilon r$ is the tangential acceleration, $\varepsilon$ is an angular acceleration, $r$ is a radius of rotation of an object, $\alpha_{r}=r \omega^{2}$ is the radial acceleration, $\omega$ is an instant angular velocity.
In classical mechanics, an angular velocity of a rotating point with acceleration is variable at the time. Expression of a tangential acceleration $\alpha_{t}$ produced at condition when a point rotates with an angular acceleration, $\varepsilon$. Expression of a radial acceleration $\alpha_{r}$ produced for an instant angular velocity, $\omega$ which a value is final for a rotating object, i.e., is constant and no an angular acceleration. However, the angular velocity of a rotating object is variable due to its angular acceleration. At condition when an angular velocity is constant, the rotating object does not have a tangential acceleration.

Eqn. (1) contains components that result on the magnitude of centrifugal and inertial forces and on the magnitude of an absolute acceleration of a rotating point.

Some textbooks remark for the calculation of acceleration the instant angular velocity of a rotating object is taken in account by the following equation:

$$
\omega=\omega_{\mathrm{m}}+\varepsilon \mathrm{t}
$$

where $\omega$ is an ultimate angular velocity, $\omega_{\text {in }}$ is an initial angular velocity, $t$ is a time interval considered, other parameters specified above.

Substituting in eqn. (2) into eqn. (1), equation of an absolute acceleration of a rotating object has the following expression:

$$
\alpha=r \sqrt{\varepsilon^{2}+\left(\omega_{i n}+\varepsilon t\right)^{4}}
$$

where $\mathrm{a}_{\mathrm{r}}=\left(\omega_{\mathrm{in}}+\varepsilon \mathrm{t}\right)^{2}$ is the radial acceleration, other parameters are as specified above.

${ }^{*}$ Corresponding author: Usubamatov R, Department of Mathematics, Kyrgyz State Technical University, Ave, Bishkek, Kyrgyzstan, Tel: +996 312545 125; E-mail: ryspek0701@yahoo.com

Received January 21, 2018; Accepted February 26, 2018; Published March 06 2018

Citation: Usubamatov R, Abdykerimova D, Dotalieva Z (2018) A Mathematical Model for an Acceleration of a Rotating Object about a Fixed Axis. J Appl Computat Math 7: 394. doi: 10.4172/2168-9679.1000394

Copyright: $\odot 2018$ Usubamatov R, et al. This is an open-access article distributed under the terms of the Creative Commons Attribution License, which permits unrestricted use, distribution, and reproduction in any medium, provided the original author and source are credited. 
However, in eqn. (3) is not correct due to unfounded mathematical approach and vagueness of intermediate conditions of acceleration analysis for the rotating object. The expression $\omega$ (in eqn. (2)) is mechanically substituted into eqn. (1), which second component derived for condition of absent the angular acceleration of rotating object $\varepsilon=0$.

A rotation of an object about an axis with an angular acceleration gives constantly increasing an angular velocity of rotation one. The magnitude of a centrifugal force and other forces will increase with increasing of an angular velocity. Incorrectness of computing for acting force magnitudes at regime of machine work reflects on the change the weight of machine components. In such case experts cannot prove the disparity of mechanisms and machines due to incorrect formula of the accelerations in textbooks, handbooks and standard methods for calculating forces acting on rotating components of machines.

Fortunately, in reality the magnitude of the radial and total acceleration is less than calculated in eqn. (3) and (4). Users of these equations calculate the acceleration that gives high magnitude and mechanisms designed on high loads and stresses than actual acting. This is the reason that mechanisms do not have failures. However, the abundant magnitude of the acceleration of machine elements leads to increase the sizes, weight and cost of machines that decrease their quality. The aforementioned factors impose a need to derive correct equations of the radial and absolute accelerations of a rotating object.

\section{Methodology}

An angular acceleration deals with a motion of a rigid object and it is the time rate of change of an angular velocity one. Figure 1 shows the link $o p$ with the radius $r$ in pure rotation, pivoted at point $o$ on the $x o y$ plane. To solve any acceleration analysis problem of a rotating point $p$ graphically, it is necessary to have only three equations: $V=\omega r$ is a linear velocity, $\alpha_{t}$ is a tangential acceleration, and $\alpha_{r}$ is a radial one of a rotating object. Acceleration analysis of the rotating object, which is subjected to a variable angular velocity is considered by corrected analytic approach. Figure 1 demonstrates two positions of the point $p$ that are separated by an angle $\alpha$. The velocity vector of the point $p$ will change along the curvature of a motion and due to rotating with a variable speed. The magnitudes of linear velocities of initial $V_{i n}$ and final $V_{f n}$ of the point $p$ are different. Hence, the velocity polygon of the rotating point $p$ is vectorially solved for these changes in the velocity, $V_{c}$.

$$
\mathrm{V}_{\mathrm{C}}=\mathrm{V}_{\text {in }}+\mathrm{V}_{\text {fn }}
$$

The vector, $V_{c}$ is the change in the linear velocity can be presented by two vectors that are radial velocity $V_{r}$ and tangential one $V_{\boldsymbol{t}}$.

$$
\mathrm{V}_{\mathrm{C}}=\mathrm{V}_{\mathrm{r}}+\mathrm{V}_{\mathrm{t}}
$$

The radial velocity vector $V_{r}$ to this curvature is directed toward the center $o$ of rotation and crosses the vector velocity $V_{f n}$.

The expression of the final variable linear velocity of a point is given by the following equation:

$$
V_{f n}=r \omega_{i n}+\varepsilon r t
$$

where $V_{\text {in }=} r \omega_{i n}, \omega_{i n}$ - an initial angular velocity of a rotating point, and $\varepsilon r t$ is an extra velocity of a moving point due to an acceleration one, other parameters of equation specified above.

For the following analysis, the radial velocity vector $V_{r}$ is represented as the sum of two vectors $V_{r . b f}$ and $V_{r . f c_{c}}$ of segments $b f$ and $f d$ respectively (Figure 1).
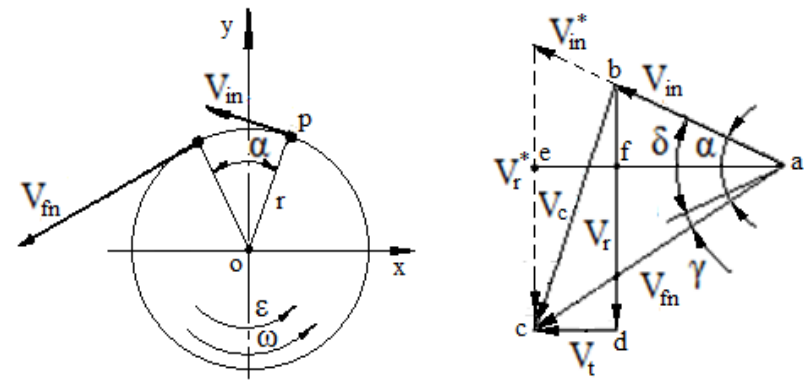

Figure 1: Velocity vectors polygon of the rotating object.

$V_{r}=V_{r . b f}+V_{r . f d}$

For following analysis is accepted a small angle $\alpha=\omega_{i n} t+\frac{\varepsilon t^{2}}{2}$ that is the sector with accelerated angular velocity of the rotating point $p$. This sector is represented by the following components: $\alpha=\delta+\gamma$, where $\delta=\omega_{\mathrm{in}} \mathrm{t}$ is the sector with initial angular velocity and $\gamma=\alpha-\delta=\frac{\varepsilon t^{2}}{2}$ is the sector with accelerated angular velocity. The two components in eqn. (7) is expressed by the following equation:

$$
\begin{aligned}
& \mathrm{V}_{r, b f}=\mathrm{V}_{\text {in }} \sin \delta / 2 \\
& \mathrm{~V}_{r . f d}=\mathrm{V}_{\mathrm{fn}} \sin (\delta / 2+\gamma)
\end{aligned}
$$

where the vector $V_{r . f d}$ is represented by the segment $f d=e c$.

Substituting in eqns. (8) and (9) into eqn. (8) yields the following equation for the radial velocity for the rotating point $p$.

$$
V_{r}=V_{\text {in }} \sin \delta / 2+V_{f n} \sin (\delta / 2+\gamma)
$$

For the small angel $\sin \Delta \varphi=\Delta \varphi$. Then, the change of the radial velocity (eqn. (10)) is represented by the following equation:

$$
\Delta V_{r}=V_{i n}(\Delta \delta / 2)+V_{f n}(\Delta \delta / 2+\Delta \gamma)
$$

Where defined above expressions of $V_{i n=} \omega_{i n} r, V_{f n=} \omega_{i n} r+\varepsilon r t$, $\Delta \delta=\omega_{\text {in }} \Delta t$, and $\Delta \gamma=\frac{\varepsilon \Delta t^{2}}{2}$ where the change in the angle depends on the change in the time. Substituting defined parameters into eqn. (11), transformation and simplification yields the following equation:

$$
\begin{aligned}
& \Delta V_{r}=\omega_{i r} r\left(\frac{\omega_{i n} \Delta t}{2}\right)+\left(\omega_{i r} r+\varepsilon t r\right)\left(\frac{\omega_{i n} \Delta t}{2}+\frac{\varepsilon \Delta t^{2}}{2}\right)=\omega_{i n} r\left(\frac{\omega_{i n} \Delta t}{2}\right)+\omega_{i n} r\left(\frac{\omega_{i n} \Delta t}{2}\right)+\omega_{i n} r\left(\frac{\varepsilon \Delta t^{2}}{2}\right)+ \\
& \operatorname{str}\left(\frac{\omega_{i n} \Delta t}{2}\right)+\varepsilon t r\left(\frac{\varepsilon \Delta t^{2}}{2}\right)
\end{aligned}
$$

In eqn. (12) is represented by differential form with respect to time

$$
\frac{d V_{r}}{d t}=\frac{\omega_{i n}^{2} r}{2} \frac{d t}{d t}+\frac{\omega_{i n}^{2} r}{2} \frac{d t}{d t}+\frac{\omega_{i n} r \varepsilon}{2} \frac{d t^{2}}{d t}+\frac{\omega_{i n} \varepsilon t r}{2} \frac{d t}{d t}+\frac{\varepsilon^{2} t r}{2} \frac{d t^{2}}{d t}
$$

where $\mathrm{dV}_{\mathrm{r}} / \mathrm{dt}=\mathrm{a}_{\mathrm{r}}$ is an expression of the radial acceleration of a rotating point at condition when an angular velocity is variable.

Solution, transformation of in eqn. (13) and simplification giving rise for the following

$$
\begin{aligned}
& a_{r}=\frac{\omega_{i n}^{2} r}{2}+\frac{\omega_{i n}^{2} r}{2}+\frac{\omega_{i n} r \varepsilon t}{4}+\frac{\omega_{i n} r \varepsilon t}{2}+\frac{r(\varepsilon t)^{2}}{4}={ }_{i n} r+ \\
& \frac{3}{4} \omega_{i n} r \varepsilon t+\frac{r(\varepsilon t)^{2}}{4}=r\left[\omega_{i n}+\frac{3}{4} \omega_{i n} \varepsilon t+\frac{(\varepsilon t)^{2}}{4}\right]
\end{aligned}
$$

where all parameters are as specified above.

The equation of the absolute acceleration of a rotating point, with 
the variable angular velocity, obtained after substituting the component of in eqn. (1) of the tangential acceleration and in eqn. (14) of the radial one into in eqn. (1). After converting this equation in algebraic form and its transformation, will have the following expression:

$$
\begin{aligned}
& a=\sqrt{(\varepsilon r)^{2}+\left\{r\left[\omega_{i n}^{2}+\frac{3}{4} \varepsilon t \omega_{i n}+\frac{(\varepsilon t)^{2}}{4}\right]\right\}^{2}}= \\
& r \sqrt{\varepsilon^{2}+\left[\omega_{i n}^{2}+\frac{3}{4} \varepsilon t \omega_{i n}+\frac{(\varepsilon t)^{2}}{4}\right]^{2}}
\end{aligned}
$$

where all parameters are as specified above.

The new equation of an absolute acceleration of a rotating point is quite different in a part of radial acceleration from the fundamental equation that represented in textbooks, (in eqn. (3)) [1-7].

\section{Case Study}

The rotating object shown in Figure 1 has the initial angular velocity of $10 \mathrm{rad} / \mathrm{s}$, accelerates at the rate of $5 \mathrm{rad} / \mathrm{s}^{2}$ and has the radius of rotation of $0.1 \mathrm{~m}$. It is necessary to determine the magnitudes of the tangential component and the radial component of accelerations and absolute one of the object after 5 seconds of rotation.

The tangential acceleration is defined by a component of eqn. (1), and the result is shown as:

$\alpha_{t=} \varepsilon r=5 \times 0.1=0.5 \mathrm{~m} / \mathrm{s}^{2}$.

The radial acceleration is defined by eqns. (13) and (3) and the results are shown as:

$$
\begin{aligned}
& a_{r}=r\left[\omega_{i n}^{2}+\frac{3}{4} \varepsilon t \omega_{i n}+\frac{(\varepsilon t)^{2}}{4}\right]=0.1\left[10^{2}+(3 / 4) \times 5 \times 5 \times 10+\right. \\
& \left.(5 \times 5)^{2} / 4\right]=44.375 \mathrm{~m} / \mathrm{s}^{2} \\
& \alpha_{r}^{*}=r\left(\omega_{i n}+\varepsilon t\right)^{2}=0.1(10+5 \times 5)^{2}=122.5 \mathrm{~m} / \mathrm{s}^{2}
\end{aligned}
$$

where $\alpha_{\mathrm{r}}^{*}$ belongs to eqn. (3).

The absolute acceleration of the rotating object defined in eqns. (14) and (3) and the results are shown as:

$$
\begin{gathered}
a=r \sqrt{\varepsilon^{2}+\left[\omega_{i n}^{2}+\frac{3}{4} \varepsilon t \omega_{i n}+\frac{(\varepsilon t)^{2}}{4}\right]^{2}}=0.1 \\
\sqrt{5^{2}+\left[10^{2}+(3 / 4) \times 5 \times 5 \times 10+\frac{(5 \times 5)^{2}}{4}\right]^{2}}=44.375 \mathrm{~m} / \mathrm{s}^{2} \\
a^{*}=r \sqrt{\varepsilon^{2}+\left(\omega_{i n}+\varepsilon t\right)^{4}}=0.1 \sqrt{5^{2}+[10+5 \times 5]^{4}}=122.501 \mathrm{~m} / \mathrm{s}^{2}
\end{gathered}
$$

where $\alpha^{*}$ belongs in eqn. (3).

The results of calculations obtained for the radial and absolute accelerations of a rotating object by new eqns. (13) and (14) and in eqns. (1) and (3) of textbooks are different. New equations of radial and absolute accelerations give an accurate result compared to those presented in the fundamental equations of textbooks, which giving a high magnitude of accelerations of a rotating point. The new mathematical model for acceleration analysis of the rotating point is common, includes correct presentation of a radial acceleration.

\section{Results and Discussion}

In engineering, a machine works with a variable velocity of rotating components that are real condition of functioning of any mechanism.
It is very important to compute an exact result of acting forces for the machine rotating components with acceleration. Computing of the dynamic force that is a product of an acceleration and mass is important for the reliable work of a machine. Analysis of eqns. (3) and (14) shows the following logical and mathematical results:

The object rotates with a constant angular velocity $\omega$ and $\varepsilon=0$. In eqns. (3) and (14) convert to the expression $a_{r}=r \omega^{2}$ that is the radial acceleration and known formula of classical mechanics.

The object starts to rotate when initial angular velocity $\omega_{\text {in }}=0$, and an angular acceleration of a rotating point is $\varepsilon$. Eqns. (3) and (14) convert to the expressions $a=r \sqrt{\varepsilon^{2}+(\varepsilon t)^{4}}$ where $a_{r}=r(\varepsilon t)^{2}$ and $a=r \sqrt{\varepsilon^{2}+\left[(\varepsilon t)^{2} / 4\right]^{2}}$ where $a_{r}=r(\varepsilon t)^{2} / 4$ respectively that have different results.

The object rotates with an initial angular velocity $\omega_{\text {in }}$ and an angular acceleration of a rotating point is $\varepsilon$. Eqns. (3) and (14) have different expressions, where the radial accelerations $a_{r}=r\left(\omega_{i n}+\varepsilon t\right)^{2}$ and $a_{r}=r\left[\omega_{i n}^{2}+\frac{3}{4} \varepsilon t \omega_{i n}+\frac{(\varepsilon t)^{2}}{4}\right]$ respectively.

In eqn. (3) of the absolute accelerations for the rotating object cannot give correct result for transient conditions of the rotating object with acceleration.

New eqn. (14) of an acceleration of the rotating object is common and can give correct result in calculation of the acceleration at any conditions, i.e., start rotation with acceleration, transient (rotation with acceleration) and stable rotation without acceleration.

The main contribution of this paper is correction of fundamental equations for acceleration for a rotating object in mechanics. The paper represents mathematical models for the radial and absolute accelerations of a rotating object giving trustworthy result in calculating. The new acceleration analysis of a rotating object can be used in educational process of kinematics and dynamics of machinery. In addition, engineers and researches for analysis of some work of machine components at transient conditions can use the new equations of accelerations of a rotating object.

\section{Conclusion}

Fundamental solution of acceleration analysis of a rotation object about an axis that represented in textbooks is not perfect and cannot give correct equations for computing radial and absolute accelerations. The main contribution of this paper is correction of fundamental equations for the acceleration for a rotating object in mechanics. The paper represents new mathematical models for the radial and absolute accelerations of a rotating object about an axis giving trustworthy result in computing. The new acceleration analysis of a rotating object should be used in textbooks of kinematics and dynamics of machinery. Also, the new equations of accelerations of a rotating object can be used by engineers and researches for analysis of machine work at transient conditions. In that regard, this is also a good example for educational processes.

\section{References}

1. Myszka DH (2004) Machines and mechanisms. Prentice Hall.

2. Syngley J, Uicker JJ (2002) Theory of Machines and Mechanisms. McGraw Hill Book Company, New York.

3. Waldron KJ, Kinzel GL, Agrawal SK (2016) Kinematics, dynamics, and design of machinery. John Wiley \& Sons. 
Citation: Usubamatov R, Abdykerimova D, Dotalieva Z (2018) A Mathematical Model for an Acceleration of a Rotating Object about a Fixed Axis. J Appl Computat Math 7: 394. doi: 10.4172/2168-9679.1000394

Page 4 of 4

4. Gregory RD (2006) Classical mechanics. Cambridge University Press.

5. Taylor JR (2005) Classical mechanics. University Science Books.

6. Hibbeler RC (2004) Engineering Mechanics Statics. Singapore: Prentice Hall.

7. Wilson CE, Sadler JP (2013) Kinematics and Dynamics of Machinery: Pearson New International Edition. Pearson Higher Ed.

8. Ferguson ES (1962) Kinematics of Mechanisms from the Time of Watt, pp: 199-209.
9. Sittel F, Müller J, Burgard W (2013) Computing velocities and accelerations from a pose time sequence in three-dimensional space. Technical Report 272 University of Freiburg, Department of Computer Science.

10. Trendel C (2006) The Kinematisch oft Vorticity. Journal of Applied Mathematics and Mechanics 37.

11. Yamada K, Asai T, Jikuya I (2016) Inverse kinematics in pyramid-type single-gimbal control moment gyro system. Journal of Guidance Control and Dynamics, pp: 1897-1907. 